\title{
Empleo de bajos salarios y pobreza en España
}

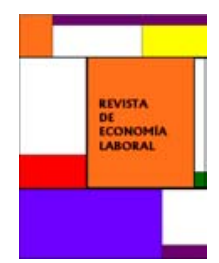

\author{
Hipólito Simón*, ${ }^{1}$, Melchor Fernández ${ }^{\dagger}$, Alberto Meixide ${ }^{\dagger}$ \\ * Universidad de Alicante e Instituto de Economía Internacional \\ $\dagger$ Universidad de Santiago de Compostela
}

Recibido el 2 de marzo de 2004; aceptado el 8 de noviembre de 2004

\section{Resumen}

La evidencia obtenida en este trabajo muestra que la percepción individual de bajos salarios es un fenómeno fuertemente asociado en España a la situación de pobreza de los hogares. Esta relación se acentúa notablemente si quien detenta el empleo de bajos salarios es el cabeza de familia del hogar, pero es significativa incluso en el caso de perceptores de rentas secundarias. La elevada falta de respuesta sobre la renta de los hogares en la Encuesta de Calidad de Vida en el Trabajo, un fenómeno habitual en las encuestas dirigidas a hogares, es una importante fuente de sesgos econométricos asociados a la selección que deben ser considerados en el análisis.

Palabras clave: Empleo de bajos salarios, pobreza, probit bivariante censurado Clasificación JEL: J31, I32.

\begin{abstract}
This paper analyses how low-wage employment, a quite relevant phenomenon in the Spanish labor market, is related to the poverty situation of Spanish households. The evidence shows that a narrow association exists between the individual perception of low wages in the Spanish labour market and the relative poverty situation of Spanish households. This relationship is reinforced if who holds the low-wage employment is the head of the family. The analysis takes into account item nonresponse in income in the Encuesta de Calidad de Vida en el Trabajo and shows that nonresponse may be an important source of selection bias.
\end{abstract}

Keywords: Low-wage employment, poverty, censored bivariate probit.

JEL Classification: J31, I32.

\footnotetext{
1 Autor para correspondencia: hsimon@ua.es. Este trabajo se ha beneficiado de la financiación procedente del proyecto de investigación GV00-081-8 de la Generalitat Valenciana. Agradecemos los comentarios y sugerencias de los participantes en el VI Encuentro de Economía Aplicada y las IV Jornadas de Economía Laboral, así como de los de dos evaluadores anónimos.
}

(c) Revista de Economía Laboral 


\section{Introducción}

El grado de desigualdad salarial de un país es en la práctica un determinante fundamental de sus diferencias internas de renta (véase, por ejemplo, Burniaux et al., 1998), y la economía española no constituye una excepción a esta influencia (Jimeno et al., 2000). Un aspecto de interés que puede permitir profundizar en el conocimiento de la relación entre el grado de diferenciación salarial y la desigualdad de la renta es el vínculo existente entre el empleo de bajos salarios y la incidencia de la pobreza. La conexión entre ambos fenómenos tiene cierto respaldo empírico en la literatura. En el plano macroeconómico, los países con una mayor incidencia del trabajo de bajos salarios presentan una mayor extensión relativa de la pobreza (Marx y Verbist, 1998, Nolan y Marx, 2000). Asimismo, si bien los trabajos que han abordado la relación entre empleo de bajos salarios y pobreza desde una perspectiva microeconómica son más escasos, sus resultados apuntan a que la percepción individual de bajos salarios está claramente asociada con la situación de pobreza de los hogares (Eurostat, 2000).

El principal objetivo de este trabajo es examinar empíricamente los efectos del empleo de bajos salarios sobre la pobreza de los hogares en España, con el fin de establecer algunos de los lazos de conexión y causalidad existentes entre la desigualdad salarial, el empleo de bajos salarios y la pobreza en nuestro país a nivel microeconómico. La relación existente entre la percepción individual de bajos salarios y la situación de pobreza del hogar del perceptor no es de partida unívoca, en la medida en que la percepción de otras rentas en el seno de la familia y la existencia en los hogares de otros perceptores potenciales de ingresos determinan que puedan darse discrepancias entre ambos fenómenos. El análisis que se aborda en el trabajo para el caso español ofrece un especial interés por varios motivos. El primero es que la estructura salarial española presenta una elevada dispersión relativa (Eurostat, 2003 y Bertola et al., 2001), de modo que el empleo de bajos salarios es un fenómeno muy extendido en nuestro mercado de trabajo, hasta el punto de que España se sitúa en términos comparativos entre los países desarrollados con una mayor incidencia relativa de este tipo de empleo (Salverda et al., 2001). El segundo es que en España se da también una elevada desigualdad de la renta (véanse Álvarez et al., 2002 y Atkinson et al., 1995) y, en consecuencia con ello, una amplia extensión de la pobreza en comparación con otros países (OCDE, 1997). Hasta donde alcanza nuestro conocimiento, 
Empleo de bajos salaries y pobreza en España

este es el primer trabajo que aborda en detalle el análisis de los efectos del empleo de bajos salarios sobre la pobreza en España ${ }^{2}$.

\section{Datos y metodología}

La base de datos microeconómicos empleada en el análisis empírico es la Encuesta de Calidad de Vida en el Trabajo (desde ahora, ECVT), una encuesta dirigida exclusivamente a individuos ocupados y elaborada por el Ministerio de Trabajo y Asuntos Sociales con periodicidad anual desde 1999. La muestra empleada en este trabajo está formada por 17.806 observaciones de las secciones cruzadas de los años que van desde 1999 hasta 2001. En el Cuadro 3 aparecen los estadísticos descriptivos de las variables.

El interés principal de este trabajo es contrastar el efecto que la percepción de un salario bajo por parte de un individuo tiene en la situación frente a la pobreza de su hogar. Con tal fin, se analizan los determinantes de la probabilidad de que un hogar sea pobre a partir de un modelo de elección discreta donde la variable dependiente es una variable ficticia que informa sobre la situación de pobreza del hogar y entre las variables explicativas se incluyen diversas características de los individuos encuestados y de sus hogares, entre ellas una variable ficticia que refleja la percepción de un salario bajo por parte del encuestado. No obstante, la ECVT es una encuesta dirigida a hogares de modo que, como es habitual en este tipo de encuestas, adolece de una elevada falta de respuesta en las variables relacionadas con los ingresos del hogar (en torno a un tercio de la muestra), a partir de las que se determina la situación de pobreza de los hogares. Aunque con mucha frecuencia en la literatura se tiende a ignorar esta cuestión, la posibilidad de la existencia de un proceso de selección no aleatorio puede conducir a estimaciones inconsistentes de los parámetros de interés. Por este motivo, el modelo de elección discreta empleado en el contraste de los factores determinantes de la pobreza es un probit bivariante censurado, que permite controlar los efectos derivados de la observabilidad parcial de la situación de pobreza de los hogares. Este modelo corrige el sesgo econométrico de selección en dos etapas à la Heckman (véase Heckman, 1978). En la primera se estima una ecuación de selección en la respuesta sobre la renta de los hogares, en la que se incluyen variables que actúan como restricciones de exclusión y permiten

\footnotetext{
2 Entre los estudios genéricos sobre la pobreza en nuestro país cabe destacar, sin ánimo de ser exhaustivos, los de Ayala et al. (1998), Cantó (1997) y Ayala y Palacio (2000). Fernández et al. (2003) analizan el empleo de bajos salarios en el mercado de trabajo español, prestando especial atención a sus determinantes.
} 
una mejor identificación del modelo. En la segunda se estima la ecuación de interés relativa a la situación de pobreza del hogar.

La información sobre ingresos salariales contenida en la ECVT está expresada en tramos de ingresos mensuales netos. Debido a esta característica de los datos no se puede utilizar la definición estándar del empleo de bajos salarios correspondiente a quienes perciben salarios inferiores a dos tercios del salario mediano de la economía. Se ha optado por considerar como perceptores de bajos salarios a aquellos asalariados con unos ingresos mensuales netos inferiores o iguales a 100.000 pesetas $^{3}$. El hecho de que la información sobre salarios en la ECVT corresponda a los salarios mensuales implica, a su vez, que el empleo de bajos salarios engloba tanto a quienes tienen salarios por hora bajos como a quienes tienen jornadas de trabajo reducidas. Dada la naturaleza del trabajo, y el hecho de que en el análisis se consideran los salarios desde la óptica de los ingresos que proporcionan a los asalariados y no como el coste salarial que suponen para el empresario, consideramos que la utilización de una definición de los salarios en términos netos y para una referencia temporal amplia es adecuada. La renta de los hogares, la variable a partir de la cual se determina la situación de pobreza de los hogares, está también expresada de forma mensual en la ECVT, lo que aconseja el uso del mismo período temporal de referencia en la definición del empleo de bajos salarios ${ }^{4}$.

La variable dependiente en la ecuación de interés del probit bivariante con observabilidad parcial es una variable binaria que refleja la situación de pobreza del hogar. Se considera que un hogar es pobre si su renta es inferior a un nivel establecido convencionalmente, la línea de la pobreza. En el cálculo de dicha línea se ha optado por utilizar un criterio relativo de pobreza y la unidad de referencia en la medición es el hogar. Frente a la alternativa de utilizar los miembros de cada hogar como ponderación en el cálculo del umbral de pobreza, se ha considerado cada hogar como una observación ${ }^{5}$. El concepto de renta es el recogido en la

\footnotetext{
${ }^{3}$ Los límites de los tramos salariales se mantienen constantes en las distintas secciones cruzadas anuales de la ECVT y no son actualizados en la encuesta.

${ }^{4}$ Se ha analizado la sensibilidad de los resultados cuando se restringe la muestra a los trabajadores a tiempo completo, con el fin de contrastar los efectos del uso de salarios mensuales sin corregir por el número de horas trabajadas. La evidencia obtenida en este caso no presenta variaciones de relevancia: los resultados del conjunto del análisis son muy parecidos para los asalariados a tiempo completo y el total de asalariados. Esta evidencia está disponible por parte de los autores ante su requerimiento.

5 Existe cierta evidencia en la literatura en el sentido de que los resultados pueden ser sensibles al uso de una ponderación alternativa en función del número de miembros del hogar, por lo que la consideración de cada hogar como una observación puede conducir a resultados distintos (una discusión del problema de las ponderaciones en la medición de
} 
ECVT, el cual no distingue entre tipos de rentas y está expresado en términos netos y en tramos de ingresos mensuales. En el tratamiento de la renta se ha optado por asignar a cada hogar el nivel de renta correspondiente al punto medio del tramo de ingresos relevante ${ }^{6}$. En la medida en que la información sobre la renta de los hogares está censurada en unos niveles máximo y mínimo de 45.000 y 750.000 pesetas, a los hogares situados en estos tramos de ingresos se les ha asignado una renta de 30.000 y 850.000 pesetas, respectivamente. Con el fin de tener en cuenta la presencia de economías de escala en el gasto, la renta del hogar ha sido ajustada en función de su tamaño a partir de una escala de equivalencia paramétrica que corresponde a un caso particular de la propuesta originalmente por Buhmann et al. (1988), con la forma genérica $R A_{i j}=R_{i} / N_{i}{ }^{\theta}$ (donde $R A_{i j}$ es la renta ajustada que se atribuye a cada miembro j del hogar i; $R_{i}$ denota la renta total sin ajustar del hogar i; $N_{i}$ el número de miembros del hogar; y $\theta$ es la escala de equivalencia), con $\theta=0,5$. En cuanto a la elección de la renta promedio, el hecho de que la información de la renta de los hogares esté censurada en sus tramos máximo y mínimo hace recomendable la utilización de la renta mediana de los hogares de la muestra, en lugar de la renta media. La ECVT está dirigida exclusivamente a hogares con al menos un ocupado, lo que implica que la muestra no es representativa del conjunto de los hogares españoles. Aunque los hogares con al menos un ocupado constituyen una proporción muy significativa de los hogares pobres en España, hasta el punto de que una proporción muy destacable de la renta disponible de estos hogares proviene de ingresos del empleo (Cantó, 1997), la incidencia de la pobreza en España es en este tipo de hogares menor que para el conjunto de la población (véase Ayala y Palacio, 2000 y Eurostat, 2000). Este hecho conduce a un más que probable sesgo al alza de las líneas de pobreza definidas a partir de la ECVT en relación con las correspondientes al total de los hogares españolas y, en consecuencia, a que la incidencia de la pobreza en nuestra muestra sobreestime la del conjunto de la población. Debido a esta circunstancia, se ha establecido el umbral de la pobreza en el $50 \%$ de la renta ajustada mediana de los hogares, un punto de corte inferior a los que se utilizan habitualmente en la literatura sobre pobreza, el $50 \%$ de la media o el $60 \%$ de la mediana. A su vez, en el trabajo se

la pobreza se encuentra en Ruiz-Huerta y Martínez, 1994).

${ }^{6}$ Este tratamiento es notablemente menos satisfactorio que la alternativa de emplear estimadores de indicadores de pobreza y desigualdad adaptados a la distribución por intervalos que presenta la renta en la ECVT (una revisión de esta literatura especializada se puede encontrar en Cowell, 2000). No obstante, y aun a pesar de las limitaciones que esta circunstancia impone en los resultados del análisis, la aplicación de estos procedimientos excede el objetivo de este trabajo. 
analiza la sensibilidad de los principales resultados ante el uso de líneas de la pobreza establecidas en otros umbrales y definidas a partir de fuentes de información alternativas a la ECVT.

\section{Resultados empíricos}

El Cuadro 1 contiene evidencia de carácter descriptivo sobre la percepción individual de bajos salarios y la situación de pobreza del hogar. La primera circunstancia a destacar es que únicamente el $23,5 \%$ de los perceptores de bajos salarios de la muestra está ubicado en hogares pobres, de modo que el grueso de este tipo de empleados corresponde a hogares que no son pobres. No obstante, la incidencia relativa de la percepción de bajos salarios es notablemente mayor en el caso de individuos ubicados en hogares pobres (del total de asalariados pertenecientes a ese tipo de hogares el $72 \%$ son perceptores de bajos salarios), que en los que lo hacen en hogares cuya renta ajustada se sitúa por encima de la línea de la pobreza (17\%). La evidencia descriptiva sugiere, pues, que, al menos en cierto sentido, empleo de bajos salarios y pobreza son fenómenos relacionados.

Cuadro 1: Distribución e incidencia del empleo de bajos salarios por nivel relativo de renta ajustada de los hogares. Fuente: ECVT 1999-2001.

\begin{tabular}{lcc}
\hline & $\begin{array}{c}\text { Distribución de los perceptores de } \\
\text { bajos salarios }\end{array}$ & $\begin{array}{c}\text { Incidencia de la percepción de bajos } \\
\text { salarios }\end{array}$ \\
\hline Total de hogares & 100 & 21,5 \\
Hogares pobres & 23,5 & 71,8 \\
Hogares no pobres & 76,5 & 17,2 \\
\hline
\end{tabular}

Nota: La distribución de los perceptores de bajos salarios mide la proporción del total de perceptores de bajos salarios en la muestra que pertenece a las categorías consideradas de hogares. La incidencia de la percepción de bajos salarios en una categoría determinada de hogares mide el porcentaje de los asalariados de la muestra ubicados en ese tipo de hogares que perciben bajos salarios. El análisis se restringe a las observaciones que presentan información sobre la renta del hogar. Se considera que un hogar es pobre si su renta ajustada se sitúa por debajo del $50 \%$ de la renta ajustada mediana de los hogares.

El Cuadro 2 contiene información sobre la incidencia de la pobreza de los hogares españoles desagregada en función del tipo de empleo del individuo encuestado. Se constata que cuando se trata de un asalariado perceptor de bajos salarios esta incidencia es comparativamente elevada. Así, mientras que el 6,6\% de los hogares españoles con al menos un ocupado es pobre ${ }^{7}$, el porcentaje se dispara al $23,5 \%$ si el ocupado es un asalariado perceptor de bajos salarios, un valor que contrasta

${ }^{7}$ Si se aplica ese porcentaje al número de ocupados en el cuarto trimestre de 2001 según la EPA, el número de ocupados "pobres" se situaría en torno a 1.064.000. 
notablemente con una tasa de pobreza de únicamente el 2,1\% para los hogares con otro tipo de asalariados. De forma general, con la excepción de los ayudas familiares sin salario, ningún tipo de empleo está, de hecho, tan relacionado con la incidencia de la pobreza en los hogares como la percepción de bajos salarios.

Cuadro 2: Tasa de pobreza de los hogares según el tipo de ocupado.

Fuente: ECVT 1999-2001.

\begin{tabular}{|c|c|c|c|c|c|}
\hline & \multicolumn{5}{|c|}{ Incidencia relativa de la pobreza (\%) } \\
\hline & \multicolumn{4}{|c|}{$\begin{array}{l}50 \% \text { renta ajustada mediana } \\
(\mathrm{ECVT})\end{array}$} & \multirow{2}{*}{$\begin{array}{c}50 \% \text { renta } \\
\text { ajustada } \\
\text { media } \\
\text { (PHOGUE) } \\
\text { Total de la } \\
\text { muestra }\end{array}$} \\
\hline & $\begin{array}{c}\text { Total de } \\
\text { la } \\
\text { muestra }\end{array}$ & $\begin{array}{c}\text { Cabeza } \\
\text { de } \\
\text { familia }\end{array}$ & $\begin{array}{l}\text { Cónyuge } \\
\text { / pareja }\end{array}$ & $\begin{array}{l}\text { Hijo/otro } \\
\text { parentesco }\end{array}$ & \\
\hline Total de ocupados & 6,6 & 7,3 & 4,2 & 6,5 & 6,2 \\
\hline Asalariados & 6,2 & 7,1 & 3,5 & 5,8 & 5,9 \\
\hline Perceptores de bajos salarios & 23,5 & 45,6 & 10,5 & 15,5 & 22,0 \\
\hline Otros asalariados & 2,1 & 2,8 & 0,4 & 1,0 & 2,1 \\
\hline Empresarios con asalariados & 3,6 & 3,3 & 1,6 & 9,5 & 3,5 \\
\hline Autónomos sin asalariados & 9,8 & 10,1 & 8,2 & 10,6 & 8,9 \\
\hline Ayuda familiar sin salario & 24,6 & 16,6 & 20,0 & 39,9 & 23,5 \\
\hline Cooperativistas & 9,9 & 10,4 & 10,0 & 7,7 & 9,6 \\
\hline
\end{tabular}

Nota: La tasa de pobreza mide la proporción de hogares con una renta ajustada por debajo de la línea de la pobreza. El análisis se restringe a las observaciones que presentan información sobre la renta del hogar en la ECVT. La renta ajustada media calculada a partir de datos del Panel de Hogares de la Unión Europea corresponde al promedio de los años 1999 a 2001 de la renta neta ajustada por unidad de consumo (escala OCDE modificada) empleando los individuos como referencia en su cálculo.

La columna derecha del Cuadro 2 recoge la incidencia de la pobreza cuando se emplea para la definición del umbral de pobreza una fuente alternativa a la ECVT, el Panel de Hogares de la Unión Europea (PHOGUE), que cubre a todo tipo de hogares y una de las líneas de la pobreza habitualmente empleadas en la literatura, el $50 \%$ de la renta media. La incidencia de la pobreza según la ECVT es, conforme cabía esperar, más elevada que según el PHOGUE, aun a pesar de que en el primer caso el umbral de la pobreza se calcula sobre la renta mediana que, en el caso de la renta ajustada, es inferior a la renta media. Esta sobreestimación general de las tasas de pobreza no parece afectar, sin embargo, a la incidencia relativa del fenómeno por tipo de empleo.

Los resultados de la estimación del probit bivariante censurado aparecen en el Cuadro 3. En la ecuación de selección, que mide la probabilidad de que el encuestado proporcione información sobre la renta del hogar y, por extensión, de que se observe su situación frente a la pobreza, la edad del encuestado y el número de miembros del hogar presentan coeficientes distintos de cero con niveles de significatividad del 
$1 \%$, mientras que el sexo del encuestado no influye significativamente. Los instrumentos empleados como restricciones de exclusión afectan en conjunto de forma significativa a la probabilidad de que se dé información sobre la renta. Así, los individuos que no responden a otras preguntas que tratan temas potencialmente confidenciales muestran una mayor propensión a no responder sobre la renta del hogar (con la única excepción de quienes no responden sobre su religión). En el mismo sentido, responden en mucha menor medida sobre la renta quienes, a juicio del encuestador, no muestran interés durante el desarrollo la entrevista. La correlación de los errores del modelo de selección y del modelo de interés, $\rho$, es positiva y distinta de cero con una significatividad del $1 \%$, por lo que los coeficientes estimados en el modelo de elección discreta con la muestra censurada sin controlar por el sesgo de selección serían sesgados e inconsistentes $^{8}$.

Según los resultados de la estimación de la ecuación de interés, el hecho de que el encuestado sea un perceptor de bajos salarios influye positiva y significativamente en la probabilidad de que su hogar de referencia sea pobre. El efecto marginal inducido por la percepción de bajos salarios es notable: que el encuestado sea un perceptor de bajos salarios aumenta un 14,6\% la probabilidad de que su hogar sea pobre frente a la alternativa de que se trate de otro tipo de asalariado. En particular, ningún otro tipo de empleo por parte de la persona de referencia induce una mayor probabilidad de pobreza del hogar. De forma más general, ninguna de las variables explicativas consideradas presenta un efecto marginal sobre la probabilidad de pobreza del hogar tan elevado como el correspondiente a la percepción de un salario bajo 9 .

\footnotetext{
${ }^{8}$ Las variables consideradas como posibles determinantes de la probabilidad de que el encuestado responda sobre la renta del hogar se han elegido en función de los resultados teóricos y empíricos de la literatura relacionada con la cuestión (véanse los trabajos de Hill y Willis, 2001, Schrapler, 2002 y Riphahn y Serfling, 2002). Estas variables, relacionadas con el desarrollo de la encuesta y la recolección de datos, no se incluyen en la ecuación de interés del modelo, por lo que actúan como restricciones de exclusión.

${ }^{9} \mathrm{El}$ impacto de las variables de control sobre la situación de pobreza se encuentra, en general, en línea con las expectativas de la literatura y con los resultados de otros estudios para España (para más detalles véanse, por ejemplo, Ayala y Palacio, 2000 y Cantó, 1997). La elección de los factores que pueden influir en la situación de pobreza de los hogares ha seguido en gran medida a los trabajos de estos autores.
} 


\section{Cuadro 3: Resultados de la estimación del probit bivariante censurado y estadísticos descriptivos de las variables. Fuente: ECVT 1999-2001.}

\begin{tabular}{|c|c|c|c|c|c|c|}
\hline \multirow[t]{3}{*}{ Variables explicativas } & \multirow[b]{3}{*}{ Med. } & \multicolumn{5}{|c|}{ Probit bivariante censurado } \\
\hline & & \multicolumn{2}{|c|}{ Ec. de selección } & \multicolumn{3}{|c|}{ Ecuación de interés } \\
\hline & & Coef. & D. E. & \multicolumn{3}{|c|}{ Coef. D. E. Ef. marg. } \\
\hline \multicolumn{7}{|l|}{ Tino de ocunado: } \\
\hline Perceptor de baios salarios & 0,172 & - & - & $1,700 * *$ & 0,06 & 0,146 \\
\hline Otro tipo de asalariado & 0,627 & - & - & \multicolumn{3}{|c|}{ Categoría base } \\
\hline No responde sobre su salario & 0,201 & & & - & - & - \\
\hline Empresario con asalariados & 0,054 & - & - & $-0,012$ & 0,13 & 0,000 \\
\hline Autónomo sin asalariados & 0,146 & - & - & $0,167^{*}$ & 0,06 & 0,004 \\
\hline Ayuda familiar sin salario & 0,008 & - & - & & 0,22 & 0,087 \\
\hline Cooperativista & 0,006 & - & - & 0,336 & 0,28 & 0,011 \\
\hline Tipo de jornada & & - & - & & & \\
\hline Tiempo completo & 0,890 & - & - & \multicolumn{3}{|c|}{ Categoría base } \\
\hline Tiempo parcial & 0,098 & - & - & $0,152^{*}$ & 0,07 & 0,004 \\
\hline No sabe & 0,012 & & & - & - & - \\
\hline \multicolumn{7}{|l|}{ Sexo } \\
\hline Mujer & 0,343 & 0,032 & 0,026 & $-0,262^{* *}$ & 0,06 & $-0,005$ \\
\hline Varón & 0,657 & & & \multicolumn{3}{|c|}{ Categoría base } \\
\hline Edad del encuestado (años) & 39,0 & $-0,010 * *$ & 0,001 & & 0,00 & 0,000 \\
\hline Tipo de hogar & & - & - & & & \\
\hline Hogar unipersonal & 0,078 & - & - & & 0,16 & 0,071 \\
\hline Familia monoparental & 0,089 & - & - & & 0,15 & 0,021 \\
\hline Otro tipo de hogar & 0,046 & - & - & $0,424^{*}$ & 0,17 & 0,014 \\
\hline Pareia sin hijos, sin otra persona en el hogar & 0,128 & - & - & \multicolumn{3}{|c|}{ Categoría base } \\
\hline Pareja sin hijos, con otra persona en el hogar & 0,011 & - & - & 0,368 & 0,23 & 0,013 \\
\hline Pareja con hijos, con otra persona en el hogar & 0,050 & - & - & 0,136 & 0,17 & 0,003 \\
\hline Pareia con hijos, sin otra persona en el hogar & 0,599 & - & - & 0,221 & 0,14 & 0,004 \\
\hline Otros perceptores de ingresos del trabajo en el hogar & & - & - & & & \\
\hline Cónyuge o pareia trabajador por cuenta ajena & 0,217 & - & - & $-1,189 * *$ & 0,10 & $-0,015$ \\
\hline Cónyuge o pareia trabajador por cuenta propia & 0,044 & - & - & $-1,041 * *$ & 0,20 & $-0,008$ \\
\hline Alguno de los hijos trabaia & 0,076 & - & - & $-0,794 * *$ & 0,12 & $-0,008$ \\
\hline Persona con otro parentesco en el hogar que & 0,123 & - & - & $-1,337 * *$ & 0,10 & $-0,010$ \\
\hline Número de miembros del hogar & 3,32 & $0,060 * *$ & 0,010 & $0,460 * *$ & 0,02 & 0,010 \\
\hline Número de miembros* Número de miembros & 12,69 & - & - & $-0,038^{* *}$ & 0,01 & $-0,001$ \\
\hline \multicolumn{7}{|l|}{ Falta de respuesta } \\
\hline No contesta sobre sus ingresos mensuales & 0,201 & $3,107 * *$ & 0,056 & - & - & - \\
\hline No contesta sobre su religión & 0,016 & $-0,139$ & 0,111 & - & - & - \\
\hline No contesta sobre su afiliación sindical & 0,043 & $0,301 * *$ & 0,064 & - & - & - \\
\hline No contesta sobre su clase social & 0,043 & $0,255 * *$ & 0,060 & - & - & - \\
\hline No contesta sobre su posicionamiento político & 0,467 & $0,240 * *$ & 0,025 & - & - & - \\
\hline \multicolumn{7}{|l|}{ Grado de interés en la entrevista } \\
\hline Muestra mucho interés en la entrevista & 0,454 & $-0,172 * *$ & 0,040 & - & - & - \\
\hline Muestra poco interés en la entrevista & 0,424 & $-0,161 * *$ & 0,040 & - & - & - \\
\hline Muestra nada de interés en la entrevista & 0,121 & \multicolumn{2}{|c|}{ Categoría base } & - & - & - \\
\hline No responde sobre la renta del hogar & 0,328 & - & - & - & - & - \\
\hline Constante & - & $0,798 * *$ & 0,068 & $-3,724 * *$ & 0,26 & - \\
\hline Número de observaciones & - & \multicolumn{2}{|c|}{17.806} & \multicolumn{3}{|c|}{17.806} \\
\hline Observaciones censuradas & & \multicolumn{2}{|c|}{-} & \multicolumn{3}{|c|}{5.835} \\
\hline Observaciones no censuradas & & \multicolumn{2}{|l|}{-} & & 11.97 & \\
\hline$\chi^{2}\left(\operatorname{Prob}>\chi^{2}\right)$ & - & $3.419,5$ & $.000)$ & & $20,4($ & $00)$ \\
\hline & - & Pseudo I & 0.425 & & $(\varrho): 0$ & $6 * *$ \\
\hline
\end{tabular}

Nota: La pobreza se corresponde a los hogares con una renta ajustada por debajo del $50 \%$ de la renta mediana ajustada. Otras variables incluidas en el modelo de elección discreta son: titulación del encuestado (9 niveles), la comunidad autónoma, el tamaño del municipio (9 estratos), ocupaciones (7) y variables del año de la observación. ${ }^{* *}$ y * indican significatividad al $1 \%$ y $5 \%$, respectivamente, según los valores de los t-ratios asintóticos. Los t-ratios se han estimado con el estimador de la varianza de Huber/White, por lo que son robustos ante la presencia de heterocedasticidad (White, 1982). 
Con el fin de contrastar la robustez de los resultados ante la definición del umbral de pobreza empleado, se ha reestimado el modelo de elección discreta empleando umbrales alternativos, el $40 \%$ y el $60 \%$ de la renta ajustada mediana según la ECVT, y el 50\% de la renta ajustada media según el PHOGUE. El coeficiente de la variable ficticia relativa a la percepción de un salario bajo es siempre positivo y estadísticamente significativo a los niveles convencionales de significatividad ${ }^{10}$. En todos los casos $\rho$ es, además, distinto de cero con una significatividad del $1 \%$, lo que corrobora la pertinencia de controlar el sesgo de selección en el proceso de estimación y, en suma, que los principales resultados son robustos a la definición del umbral de pobreza.

Por último, un aspecto que puede determinar la relación entre la percepción individual de bajos salarios y la pobreza de los hogares es la posición que ocupan en el hogar los perceptores de este tipo de salarios. Aunque la percepción de bajos salarios se distribuye de una forma relativamente similar entre los distintos miembros de los hogares (el $30,8 \%$ de los perceptores de bajos salarios son cabeza de familia, el $28,8 \%$ cónyuges o pareja y el 40,4\% son hijos o tienen otro parentesco), los cabeza de familia sufren una menor incidencia del empleo de bajos salarios $(10,5 \%)$ que sus parejas u otros miembros del hogar $(35,9 \%$ y $36 \%$, respectivamente). La existencia de una relación mucho más estrecha entre la percepción de bajos salarios y la pobreza en el caso de que los perceptores de aquéllos sean cabeza de familia es confirmada por los resultados empíricos. Así, mientras que la incidencia de la pobreza es del $45,6 \%$ en el caso de que el perceptor de bajos salarios en el hogar sea el cabeza de familia, se sitúa entre el 10,5\% y el $15,5 \%$ en caso de que sea un perceptor de rentas secundarias (Cuadro 2). En el mismo sentido, se constata que el efecto marginal de la percepción de bajos salarios sobre la probabilidad de pobreza del hogar es sustancialmente mayor en el caso de que el perceptor de bajos salarios sea cabeza de familia ${ }^{11}$.

\section{Conclusiones}

Los resultados obtenidos en este trabajo muestran que la percepción individual de bajos salarios influye significativamente en la

\footnotetext{
${ }_{10}$ Los resultados completos de estas estimaciones no se han incluido en el trabajo por una cuestión de espacio. Los mismos están disponibles por parte de los autores ante su requerimiento.

11 Los resultados completos de las estimaciones desagregadas del probit bivariante censurado por tipo de parentesco no se han incluido en el trabajo por una cuestión de espacio. Los mismos están disponibles por parte de los autores ante su requerimiento.
} 
situación de pobreza de los hogares españoles. La asociación entre ambos fenómenos es más intensa si el perceptor de bajos salarios es el cabeza de familia del hogar, pero es significativa incluso cuando el mismo es un perceptor de rentas secundarias. Esta evidencia apunta, en suma, a que la estructura salarial comparativamente dispersa que presenta el mercado de trabajo español, y que se manifiesta en una elevada incidencia relativa del empleo de bajos salarios, influye notablemente en el patrón de incidencia de la pobreza en nuestro país. Por otra parte, una hipotética estrategia de política económica y social que tuviese como objetivo la reducción de la pobreza a través de la disminución del empleo de bajos salarios debería centrarse en los casos en los que este fenómeno afecta al cabeza de familia, aunque cabe considerar que, en la medida en que el grueso de los perceptores de bajos salarios no se ubica en hogares pobres, beneficiaría fundamentalmente a hogares que no se encuentran en situaciones de pobreza.

\section{Bibliografía}

Álvarez, S.; Prieto, J. y R. Salas (2002): "The Evolution of Income Inequality in the European Union during the Period 1993-96", Instituto de Estudios Fiscales, Papeles de Trabajo 2002-10.

Atkinson, A.B.; Rainwater, L. y T. Smeeding (1995): Income Distribution in OECD Countries, OCDE, Paris.

Ayala, L; Esteve, F.; García Lizana, A.; Muñoz de Bustillo, R.; Renes, V.; y R. Rodríguez Cabrero (1998): Las condiciones de vida de la población pobre en España, Fundación FOESSA, Madrid.

Ayala, L. y J.I. Palacio (2000): "Hogares de baja renta en España: caracterización y determinantes", Revista de Economía Aplicada, vol. 8 (23), 35-70.

Bertola, G., Blau, F. y L. Kahn (2001): "Comparative Analysis of Labor Market Outcomes: Lessons for the US from International Long-Run Evidence", NBER working paper 8526.

Buhmann, B.; Rainwater, L.; Schmaus, G. y T. Smeeding (1988): "Equivalence scales, well-being, inequality, and poverty: sensitivity 
estimates across ten countries using the Luxembourg Income Study (LIS) database", Review of Income and Wealth, 34, 115-142.

Burniaux, J.M.; Dang, T.T.; Fore, D.; Forster, M. y M. Mira (1998): "Income Distribution and Poverty in Selected Oecd Countries", OECD Economics Department working paper 189, OCDE, Paris.

Cantó, O. (1997): "Desempleo y pobreza en la España de los 90", Papeles de economía española, 72, 88-108.

Cowell, F.A. (2000): "Measurement of Inequality", en A.B. Atkinson y F. Bourguignon (eds.) Handbook of Income Distribution, vol. I, editorial North-Holland, Amsterdam, 87-166.

Eurostat (2000): "Low-wage employees in EU countries", Statistics in Focus, 11.

Eurostat (2003): Employment in Europe 2003, Oficina de Publicaciones de las Comunidades Europeas, Luxemburgo.

Fernández, M.; Meixide, M. y H. Simón (2003): "El trabajo de bajos salarios en España”, Estudio sobre la economía española $n^{\circ} 152$, FEDEA, Madrid.

Heckman, J. (1978): "Dummy Endogenous Variables in a Simultaneous Equation System”, Econometrica, 46(4), 931-959.

Hill, D. y R. Willis (2001): "Reducing panel attrition: a search for effective policy instruments", Journal of Human Resources, 36 (3), 416-438.

Jimeno, J.F.; Cantó, O.; Cardoso, A.R.; Izquierdo, M. y C. Farinha (2000): "Integration and Inequality: Lessons from the Accessions of Portugal and Spain to the EU”, documento de trabajo 2000-10, FEDEA, Madrid.

Marx, I. y G. Verbist (1998): "Low-Paid Work and Poverty: A CrossCountry Perspective",en S. Bazen, M. Gregory y W. Salverda (eds.) Low Wage Employment in Europe, editorial Edwar Elgar, 63-86.

Nolan, B. y I. Marx (2000): "Low Pay and Household Poverty", en Gregory, M., Salverda, W. y S. Bazen, Low pay: an analytical perspective, Oxford University Press, 100-119. 
OCDE (1997): "Labour market policies: New challenges policies for lowpaid workers and unskilled job seekers", GD[97]160, OCDE, París.

Riphahn, R.T. y O. Serfling (2002): "Item non-response on income and wealth questions", IZA discussion paper $n^{0} 573$.

Ruiz-Huerta, J. y R. Martínez (1994): "La pobreza en España: ¿qué nos muestran las Encuestas de Presupuestos Familiares?", Documentación Social, 96, 15-110.

Schrapler, J.P. (2002): "Respondent Behavior in Panel Studies-A Case Study for Income-Nonresponse by means of the German Socio-Economic Panel (GSOEP)", German Institute for Economic Research discussion paper 299.

Salverda, W.; Bazen, S. y M. Gregory (coord.) (2001): "The EuropeanAmerican Employment Gap, Wage Inequality, Earnings Mobility and Skill: A Study for France, Germany, the Netherlands, the United Kingdom and the United States", informe para la Comisión Europea, Dirección General de Empleo y Asuntos Sociales, Madrid.

White, H. (1982): "Maximum Likelihood Estimation of Misspecified Models", Econometrica, 50, 1-25. 